\title{
Interface controlled growth of nanostructures in discontinuous Ag and Au thin films fabricated by ion beam sputter deposition for plasmonic applications
}

\author{
R BRAHMA and M GHANASHYAM KRISHNA* \\ School of Physics, University of Hyderabad, Central University PO, Hyderabad 500 046, India
}

MS received 28 March 2012

\begin{abstract}
The growth of discontinuous thin films of Ag and Au by low energy ion beam sputter deposition is reported. The study focuses on the role of the film-substrate in determining the shape and size of nanostructures achieved in such films. Ag films were deposited using Ar ion energy of $150 \mathrm{eV}$ while the Au films were deposited with Ar ion energies of 250-450 eV. Three types of interfaces were investigated in this study. The first set of film-substrate interfaces consisted of $\mathrm{Ag}$ and Au films grown on borosilicate glass and carbon coated Cu grids used as substrates. The second set of films was metallic bilayers in which one of the metals (Ag or Au) was grown on a continuous film of the other metal (Au or Ag). The third set of interfaces comprised of discontinuous Ag and Au films deposited on different dielectrics such as $\mathrm{SiO}_{2}, \mathrm{TiO}_{2}$ and $\mathrm{ZrO}_{2}$. In each case, a rich variety of nanostructures including self organized arrays of nanoparticles, nanoclusters and nanoneedles have been achieved. The role of the film-substrate interface is discussed within the framework of existing theories of thin film nucleation and growth. Interfacial nanostructuring of thin films is demonstrated to be a viable technique to realize a variety of nanostructures. The use of interfacial nanostructuring for plasmonic applications is demonstrated. It is shown that the surface Plasmon resonance of the metal nanostructures can be tuned over a wide range of wavelengths from 400 to $700 \mathrm{~nm}$ by controlling the film-substrate interface.
\end{abstract}

Keywords. Ion beam sputter deposition; discontinuous thin films; Ag; Au; film-substrate interface.

\section{Introduction}

Two approaches are normally followed to achieve nanostructures in thin film form. The first set of techniques is based on lithographic techniques such as electron beam lithography, nanoimprint technology (Balla et al 2008; Tennant and Bleier 2011). The second set of techniques is based on nonlithographic and direct writing methods such as focussed ion beam, dip pen lithography, laser shock processing, electric field induced nanostructuring etc (Gamo 1997; Krishna and Kumar 2009). Both these sets of approaches are postprocessing methods that achieve nanostructures after a film is deposited on a substrate.

A third approach that has been successfully used for realizing a wide range of nanostructures are those based on soft chemical processes leading to self-organized, self-ordered and self-assembled nanostructures (Masala and Seshadri 2004; Zhang and Wang 2009; Rao et al 2006; Didiot et al 2007). However, studies on the use of physical vapour deposition (PVD) techniques to fabricate such structures are sparse (Teichert 2002; Woll et al 2002; Stangl et al 2004). Ion beam sputter deposition (IBSD) is a PVD technique that

\footnotetext{
*Author for correspondence (mgksp@uohyd.ernet.in)
}

has the ability to produce nanostructured thin films at ambient temperature without the need for any post-deposition processing. This is in contrast to sol-gel techniques that require extensive post-deposition processing to achieve the same kind of nanostructures. This makes IBSD more compatible to the semiconductor and electronics industries and is hence the preferred technique in the current study.

There are generally four main steps in the evolution of thin films deposited on substrates by PVD (Maissel and Glang 1970). These are nucleation, island growth, agglomeration and finally continuous film. The first two stages would lead to largely discontinuous thin films. The microstructure at these early stages of growth would be controlled by process parameters like rates of deposition and substrate temperature and most importantly the nature of the film-substrate interface. For constant temperature and rate of deposition the growth would entirely be dependent on the nature of film-substrate interface and the thermodynamics in the early stages of thin film growth. The thermodynamics involves the minimization and control of the total free energy of the film-substrate interface. This is the basic premise of the current work and it is demonstrated that interfacial nanostructuring is a simple approach to achieve a variety of nanostructures such as nanoclusters, nanoparticle arrays and nanoneedles. The other motivation of the current work is to investigate the use of discontinuous and nansostructured thin films for plasmonic applications. It is demonstrated that the films grown on 
different substrates exhibit a range of tenability that depends entirely on the film-substrate interface.

The morphological evolution of thin films (3-50 nm thickness) of Ag and Au deposited on borosilicate glass and carbon coated $\mathrm{Cu}$ grid (used for TEM) substrates by ion beam sputter deposition is reported. In addition, $\mathrm{Ag} / \mathrm{Au}, \mathrm{Au} / \mathrm{Ag}$ and $\mathrm{Ag}$ or $\mathrm{Au} /$ dielectric bilayers grown on borosilicate glass and fused silica substrates are also investigated. Ag films were deposited at an Ar ion beam energy of $150 \mathrm{eV}$ whereas the $\mathrm{Au}$ films were deposited at an ion energy of 250 to $450 \mathrm{eV}$. The optical response of the films in the wavelength range from 190 to $2500 \mathrm{~nm}$ is also reported.

\section{Experimental}

Ag (99.99\% purity) and Au (99.9\% purity) targets were sputtered using a Kaufman type ion source (DC25, Oxford Applied Research, UK) with Argon ions of energy in the range of 150 to $450 \mathrm{eV}$ and beam currents of 4-6 mA. The target substrate distance was about $14 \mathrm{~cm}$ and substrates were not heated during deposition. The combination of low energy, low flux and large substrate distances resulted in extremely good control over the microstructural evolution of the Ag and Au films (Brahma and Krishna 2008, 2011). The microstructure of all the films was examined in an Atomic Force microscope (Model SPA 400, SII Inc., Japan) operating in the dynamic force mode. Transmission electron micrographs were obtained in a transmission electron microscope (Tecnai 20 G2 STwin, FEI, The Netherlands) operating at $200 \mathrm{kV}$. Electron diffraction patterns (EDPs) were recorded with a Gatan CCD camera. A $10 \mathrm{~nm}$ gold film deposited on the grid was used for camera length calibration purposes. Scanning electron microscope images were obtained in a Carl Zeiss Model Ultra55 microscope.

It may be noted that thicknesses for only the continuous films were measurable. The thickness of these continuous films was measured using a stylus profilometer (Model XP-1, Ambios Technology, USA). From the total duration of deposition and measured thickness, the rate of depositon was estimated to vary from 0.01 to $0.1 \mathrm{~nm} / \mathrm{s}$, with increase in ion energy. For all the discontinuous films, only the duration of deposition was mentioned since thickness was not measurable. However, an estimate of thickness in these cases is possible since rate of deposition is calibrated.

The following sets of films were studied in the current work: (i) single layer Ag and Au films on borosilicate glass (BSG) and carbon coated grid (CCG) substrates, (ii) bilayers consisting of $\mathrm{Ag}$ or $\mathrm{Au}$ as top layer and $\mathrm{Au}$ or $\mathrm{Ag}$ as the bottom layer; referred to as metal-metal bilayers and (iii) bilayers consisting of $\mathrm{Ag}$ or $\mathrm{Au}$ as top layer and $\mathrm{SiO}_{2}, \mathrm{TiO}_{2}$ or $\mathrm{ZrO}_{2}$ as the bottom layer, referred to as metal-dielectric bilayers.

The Ag and Au films on all these surfaces were grown in the same run so that all other deposition parameters are the same.

\section{Results and discussion}

The morphology of Ag and Au films deposited on to BSG substrates are shown in figures 1(a) and (b). The Ag films (figure 1(a)) were deposited on to BSG substrate at $150 \mathrm{eV}$ whereas the Au films were deposited at $250 \mathrm{eV}$ (figure 1(b)). The reason for this difference in energy is that the sputter yield of $\mathrm{Au}$ is much lower than that for Ag. Hence, no film deposition occurred at $150 \mathrm{eV}$ in the case of $\mathrm{Au}$ even for a duration of $40 \mathrm{~min}$. At $250 \mathrm{eV}$, after sputtering for $25 \mathrm{~min}$, there was initial evidence for film deposition on BSG substrates, as is evident from figure 1(b). The observed behaviour can be attributed to two factors viz. low rate of deposition and high contact angle. To distinguish between the contributions, each of these factors, Au films, were deposited on carbon coated $\mathrm{Cu}$ grids (CCG) at the same beam energy. However, it was found that the Au films did not exhibit any observable growth on CCG for energies up to $450 \mathrm{eV}$, clearly indicating that the interfacial energy dominated over the low rate of deposition. This is inferred from the fact that the rate of deposition increases with increase in ion energy and, therefore, should increase the coverage of the substrate surface with duration of deposition. "Potato" shaped cluster of particles appeared when the films were deposited on CCG at an ion energy of $450 \mathrm{eV}$ and for a duration of $40 \mathrm{~min}$ as shown in figure 2(a). For comparison, TEM image of an Ag film grown for 10 min directly onto CCG at $150 \mathrm{eV}$ is shown in figure 2(b). It is evident from this figure that Ag films have started nucleating as in the case of the films grown on the BSG substrates. In both cases, Ag and $\mathrm{Au}$ deposited on $\mathrm{CCG}$, the particles arrange themselves in the form of array with sizes between 5 and $20 \mathrm{~nm}$. Therefore, it can be inferred that the interfacial energy of Au on $\mathrm{BSG}$ is lower than that for Au on CCG leading to differences in growth on the two substrates. The high resolution TEM images displayed in figures 2(a) and (b) and the selected area electron diffraction patterns shown in figures 2(c) and (d) for $\mathrm{Au}$ and Ag, respectively indicate that the films are crystalline.

To investigate the growth patterns on a scale larger than those available with AFM and TEM, the growth was also examined under a scanning electron microscope. The early stages of growth on BSG substrates for both Ag $(150 \mathrm{eV}$, $10 \mathrm{~min}$ ) and $\mathrm{Au}$ (250 eV, $20 \mathrm{~min})$ are similar, as seen from figures 3(a) and (b), respectively. The microstructure consists of an organized array of nanoparticles, as seen in the AFM and TEM images. Interestingly, in both cases the formation of nanoneedles has also been observed, as shown in figures 3(c) and (d), respectively for Ag and Au. They are of $500 \mathrm{~nm}$ in length with diameters of the order of 30-40 nm. Interestingly in the case of $\mathrm{Ag}$, the nanoneedle formation follows the process of organization into an array of nanoparticles while it precedes it in the case of Au. The reasons for this difference in behaviour are not clear.

Another set of interfaces investigated were the metalmetal interfaces consisting of (i) a fixed thickness of $\mathrm{Ag}$ onto which $\mathrm{Au}$ of different thicknesses were grown (these are referred to as $\mathrm{Ag} / \mathrm{Au}$ bilayers) and (ii) a fixed thickness of $\mathrm{Au}$ 

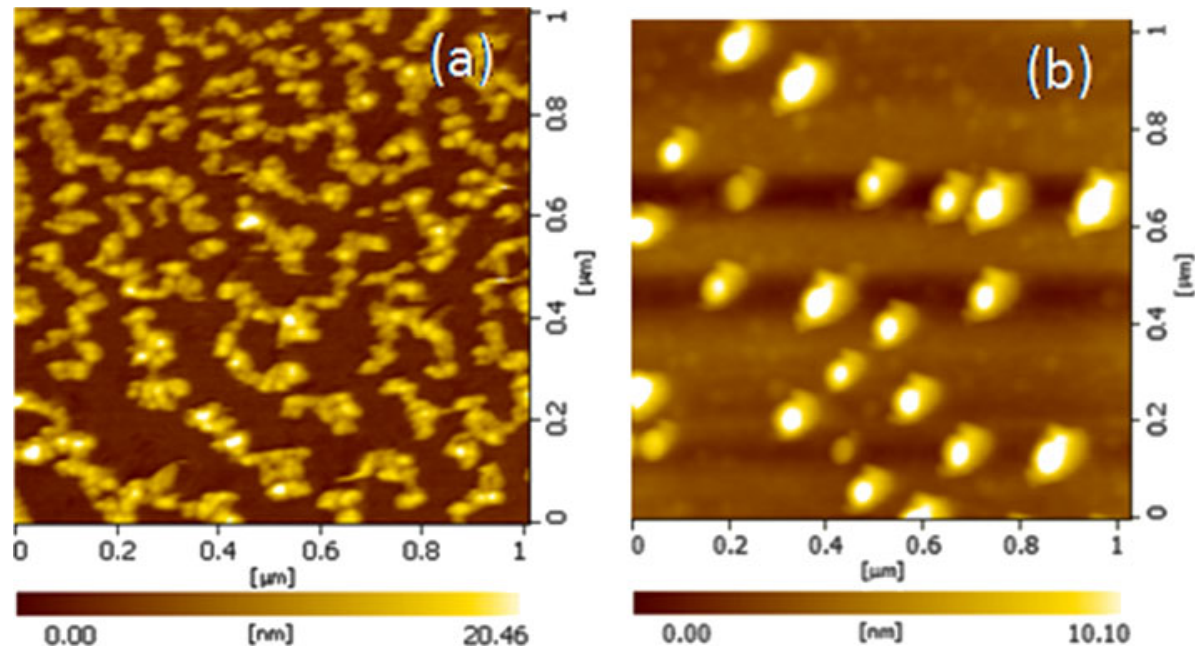

Figure 1. Atomic force microscope images of (a) Ag film deposited using an Ar ion energy of $150 \mathrm{eV}$ for $15 \mathrm{~min}$ and (b) Au film deposited using an Ar ion energy of $250 \mathrm{eV}$ for $25 \mathrm{~min}$ onto BSG substrates.
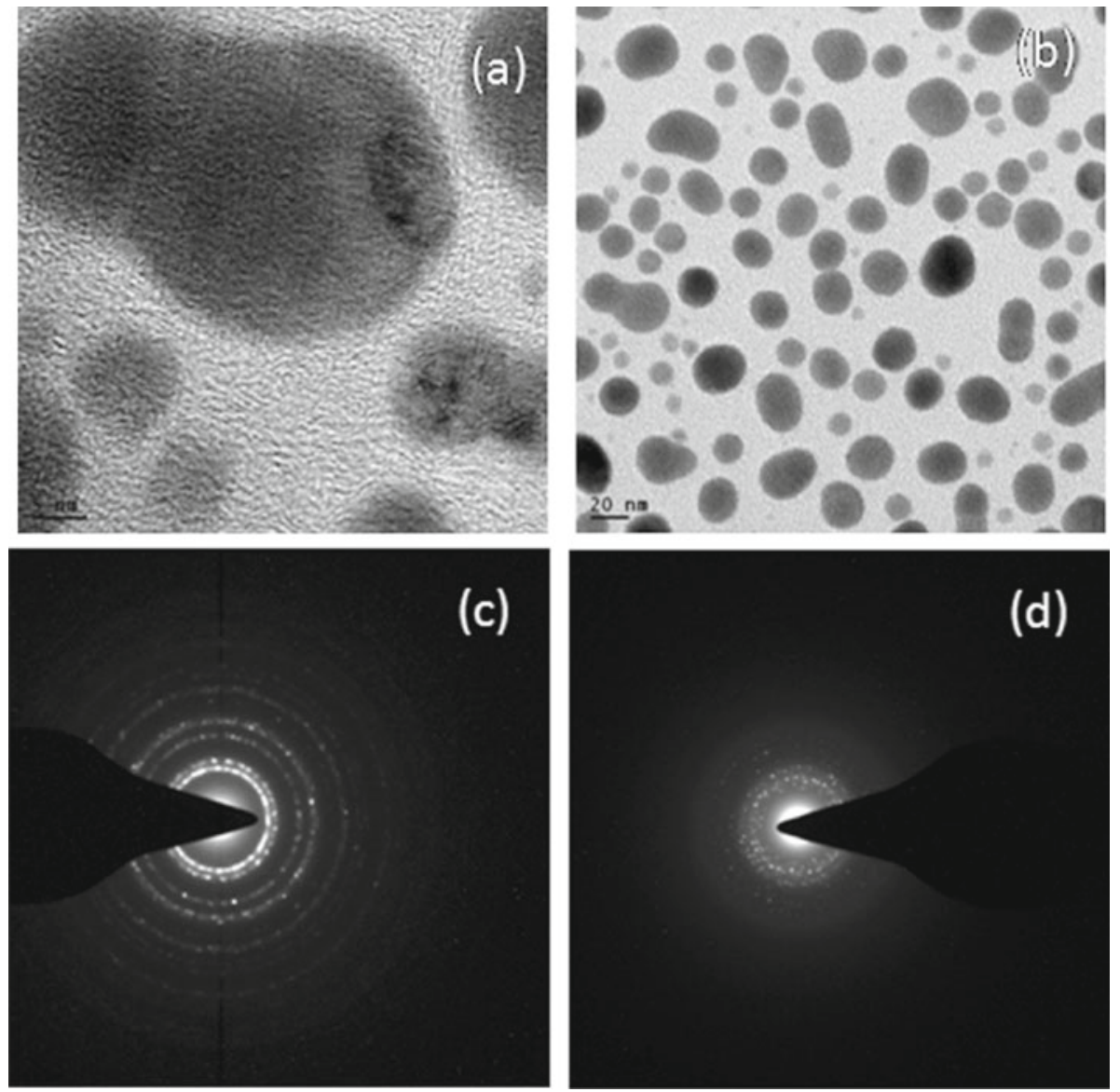

Figure 2. Transmission electron microscope images of (a) Au film deposited using an Ar ion energy of $450 \mathrm{eV}$ for $40 \mathrm{~min}$ and (b) Ag film deposited using an Ar ion energy of $150 \mathrm{eV}$ for $10 \mathrm{~min}$ onto carbon coated $\mathrm{Cu}$ grids used as substrates. The corresponding selected area diffraction patterns are shown in figures $2(\mathbf{c})$ and (d), respectively. 

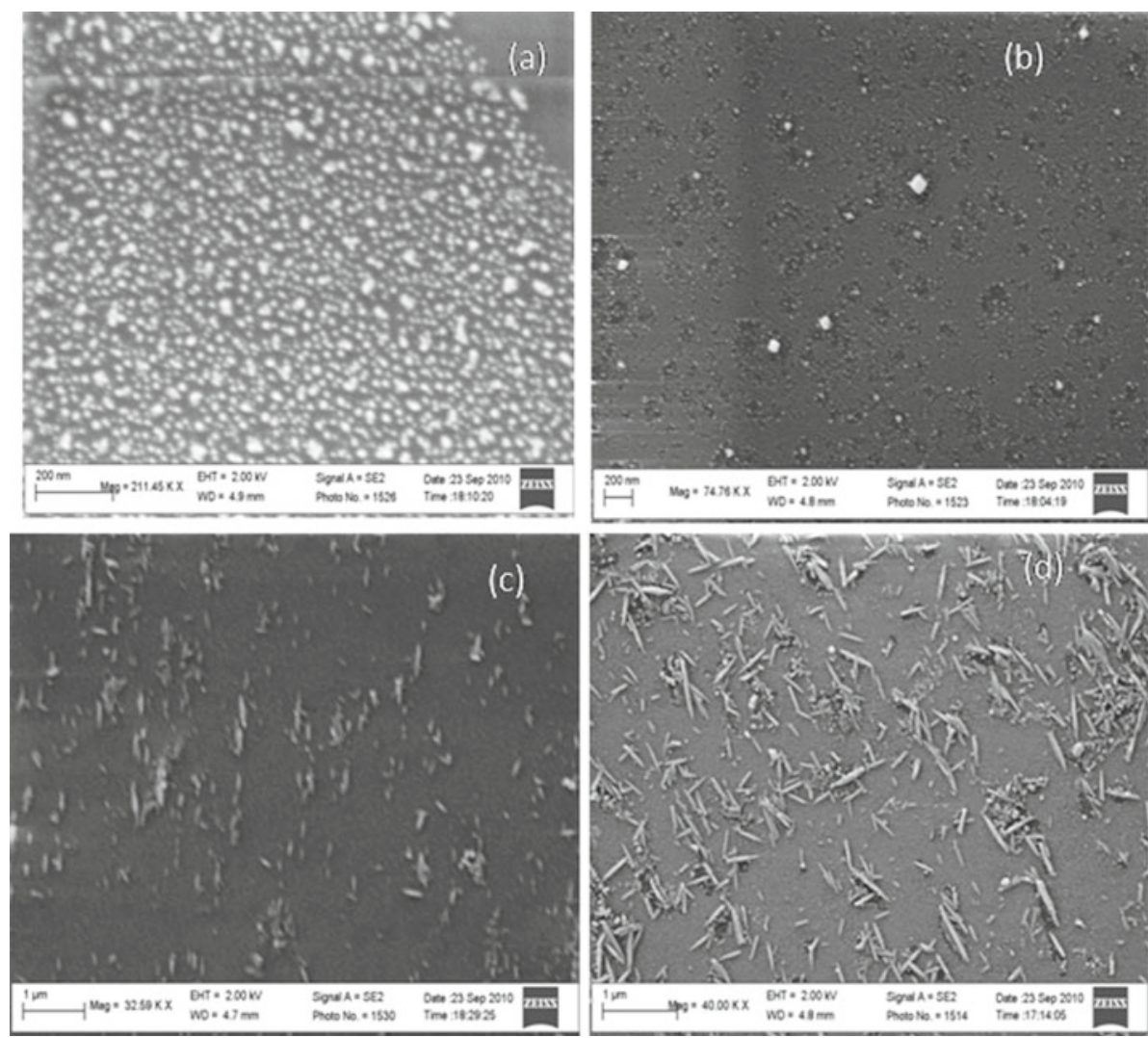

Figure 3. Scanning electron microscope images of (a) Ag film deposited using an Ar ion energy of $150 \mathrm{eV}$ for $30 \mathrm{~min}$, (b) Au film deposited using an Ar ion energy of $250 \mathrm{eV}$ for 20 min onto (c) Ag film deposited using an Ar ion energy of $150 \mathrm{eV}$ for $20 \mathrm{~min}$ and (d) Au film deposited using an Ar ion energy of $250 \mathrm{eV}$ for 30 min onto BSG substrates.

onto which $\mathrm{Ag}$ of different thicknesses were grown (these are referred to as $\mathrm{Ag} / \mathrm{Au}$ bilayers). The deposition sequence for fabricating the bilayers was: (I) deposit a continuous layer of $\mathrm{Au}$ or $\mathrm{Ag}$ onto BSG substrate and (II) deposit $\mathrm{Ag}$ or $\mathrm{Au}$ layers onto the lower layers for different durations.

The Ag films were deposited using an Ar ion energy of $150 \mathrm{eV}$ whereas the Au films were deposited using an ion energy of $250 \mathrm{eV}$.

The morphology of a continuous Ag film of $30 \mathrm{~nm}$ thickness is shown in figure 4(a). It is observed that the films are continuous and densely packed with nanoclustered spherical particles of sizes between 30 and $50 \mathrm{~nm}$. The morphology of the $\mathrm{Ag} / \mathrm{Au}$ bilayers is shown in figures 4(b) and (c). It is evident that after $10 \mathrm{~min}$ of deposition of $\mathrm{Au}$ on $\mathrm{Ag}$, (figure 4(b)), the surface of Ag has already been wetted by the $\mathrm{Au}$ particles which form a dense network of spherical nanoclusters of 30-50 nm diameter. The Au film deposited onto the Ag layer for $30 \mathrm{~min}$ is continuous with a dense microstructure and very low surface roughness, as displayed in figure 4(c). This behaviour is in contrast to that observed for Au films deposited on BSG and CCG, discussed earlier, clearly demonstrating the role of the film-substrate interface.

The growth of $\mathrm{Ag}$ on a continuous Au layer is shown in figures 5(a) to (c). The Au film is continuous and dense with a thickness of $50 \mathrm{~nm}$ (figure 5(a)). The morphology of the Ag film deposited at $150 \mathrm{eV}$ for $20 \mathrm{~min}$ is clearly discontinuous (figure 5(b)) and consists of a randomly distributed array of nanoparticles. The discontinuous microstructure is retained even after a deposition duration of $45 \mathrm{~min}$, as shown in figure 5(c). This is in contrast to the morphological evolution of Au on Ag (shown in figures 4(b) and (c)) where continuous and dense Au films were observed for much shorter durations of deposition.

The third set of films investigated were $\mathrm{Ag}$ and $\mathrm{Au}$ films deposited on $\mathrm{SiO}_{2}, \mathrm{TiO}_{2}$ and $\mathrm{ZrO}_{2}$ substrates. The $\mathrm{TiO}_{2}$ and $\mathrm{ZrO}_{2}$ surfaces were obtained by the sol-gel technique described elsewhere (Brahma and Krishna 2011), while the $\mathrm{SiO}_{2}$ surface was fused silica substrate. The morphology of the $\mathrm{Ag}$ films on these surfaces is shown in figures 6(a) to (c) whereas that for Au films on the same surfaces is shown in figures 7(a) to (c). In both cases, it is observed that the substrate surface has a profound effect on the film grown on top. Ag films are discontinuous on $\mathrm{SiO}_{2}$ and $\mathrm{TiO}_{2}$ while they are continuous on $\mathrm{ZrO}_{2}$. In contrast, $\mathrm{Au}$ films are continuous on $\mathrm{SiO}_{2}$ and $\mathrm{TiO}_{2}$ while they are discontinuous on $\mathrm{ZrO}_{2}$, reinforcing the observation made about the significance of contact angle and therefore, film-substrate in the previous cases of single layers and metal-metal bilayers.

The effect of these morphological variations on the surface plasmon resonance behaviour of the nanostructured films is 

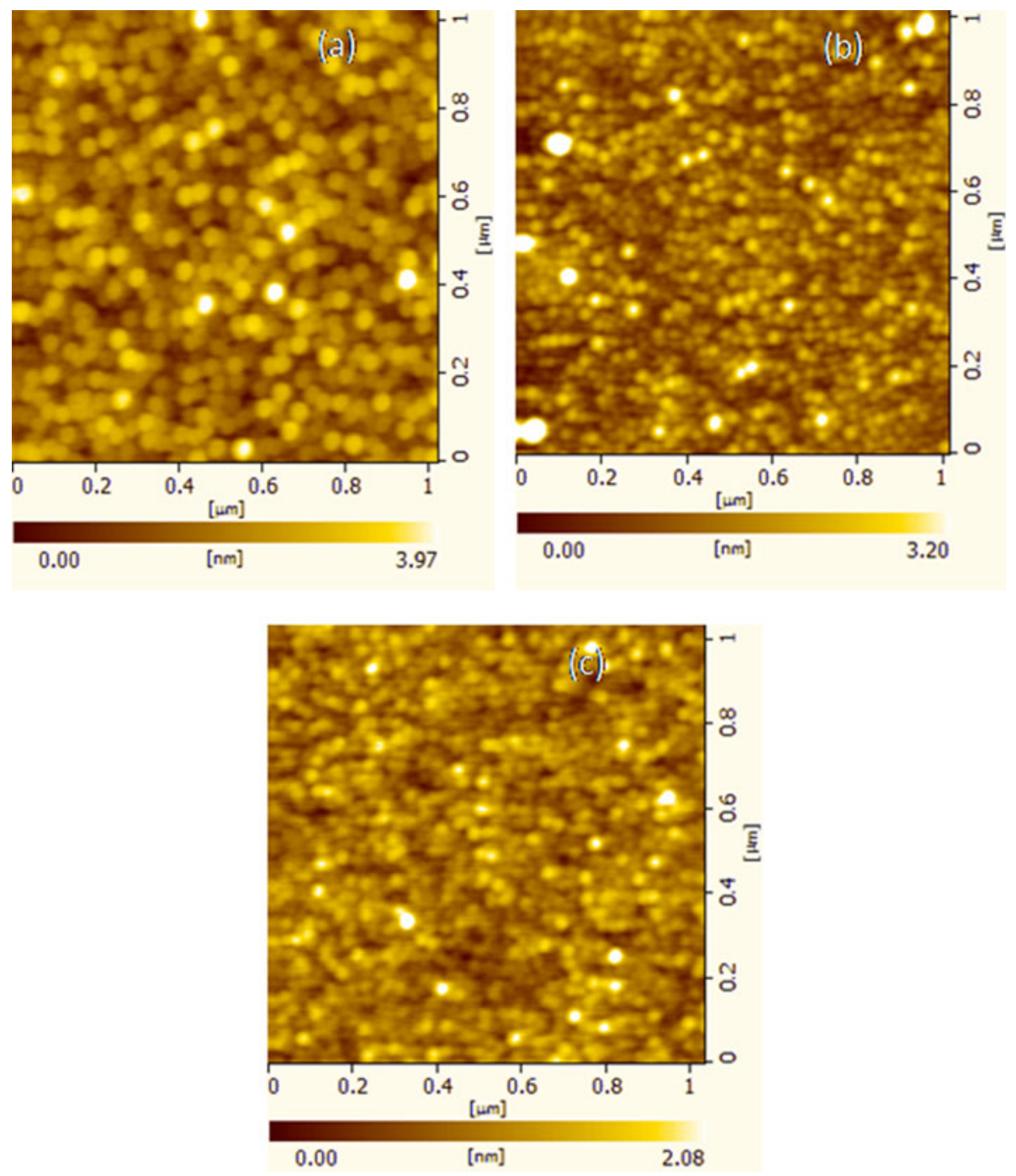

Figure 4. Atomic force images of (a) $30 \mathrm{~nm}$ thick continuous Ag film deposited onto BSG substrate and $\mathrm{Ag} / \mathrm{Au}$ bilayer consisting of $\mathrm{Ag}$ film shown in (a) and an overlayer of Au deposited on top using an Ar ion energy of $250 \mathrm{eV}$ for (b) 10 and (c) $30 \mathrm{~min}$.

shown in figures 8 and 9. The absorption spectra of single layer Ag films, as a function increasing time of deposition on BSG substrates is shown in figure 8(a). It is observed that the surface plasmon resonance peak shifts from $380-580 \mathrm{~nm}$ as the duration increases from $10-30 \mathrm{~min}$. The absorption behaviour for $\mathrm{Au}$ films on BSG substrates for increasing durations of deposition and fixed duration with increase in ion energy, displayed in figure 8(b) shows limited tunability of plasmon resonance peak from 558 to $584 \mathrm{~nm}$. However, when the Ag films are deposited on top of a continuous Au layer they show tunability of plasmon resonance over a larger range of wavelengths extending from 530-696 nm (figure 8(c)). A similar behaviour is observed in the case of $\mathrm{Au}$ films on $\mathrm{Ag}$ surfaces as seen in figure 8(d). The plasmon resonance peak position on dielectric surfaces is dependent on the refractive index of the dielectric layer underneath. This is shown in figures 9(a) and (b) for $\mathrm{Ag}$ and $\mathrm{Au}$, respectively. The resonances occur at 420, 428 and $440 \mathrm{~nm}$ for $\mathrm{Ag}$ on $\mathrm{SiO}_{2}, \mathrm{ZrO}_{2}$ and $\mathrm{TiO}_{2}$, respectively. For Au films the peaks occur at 606, 630 and $634 \mathrm{~nm}$, clearly showing very limited tunability. Two observations that can be made from the results presented above are that (i) the position of plasmon resonance is dependent on the nature of the substrate and therefore, morphology of the films, i.e. particle size, shape and separation and (ii) the tunability of plasmon resonance is also very sensitive to the nature of the film-substrate interface. Precise control of the film-substrate interactions leads to a very large range of plasmon resonances that are useful for a wide variety of applications.

Siegel et al (2011) studied the formation of discontinuous gold nanostructures by sputter deposition onto glass substrates. They have also seen the formation of an array of Au nanoparticles in their discontinuous films. Beszeda et al (2006) have shown that the continuous Au films deposited on $\mathrm{SrTiO}_{3}$ substrates acquire a discontinuous beaded microstructure, similar to those observed in the current 

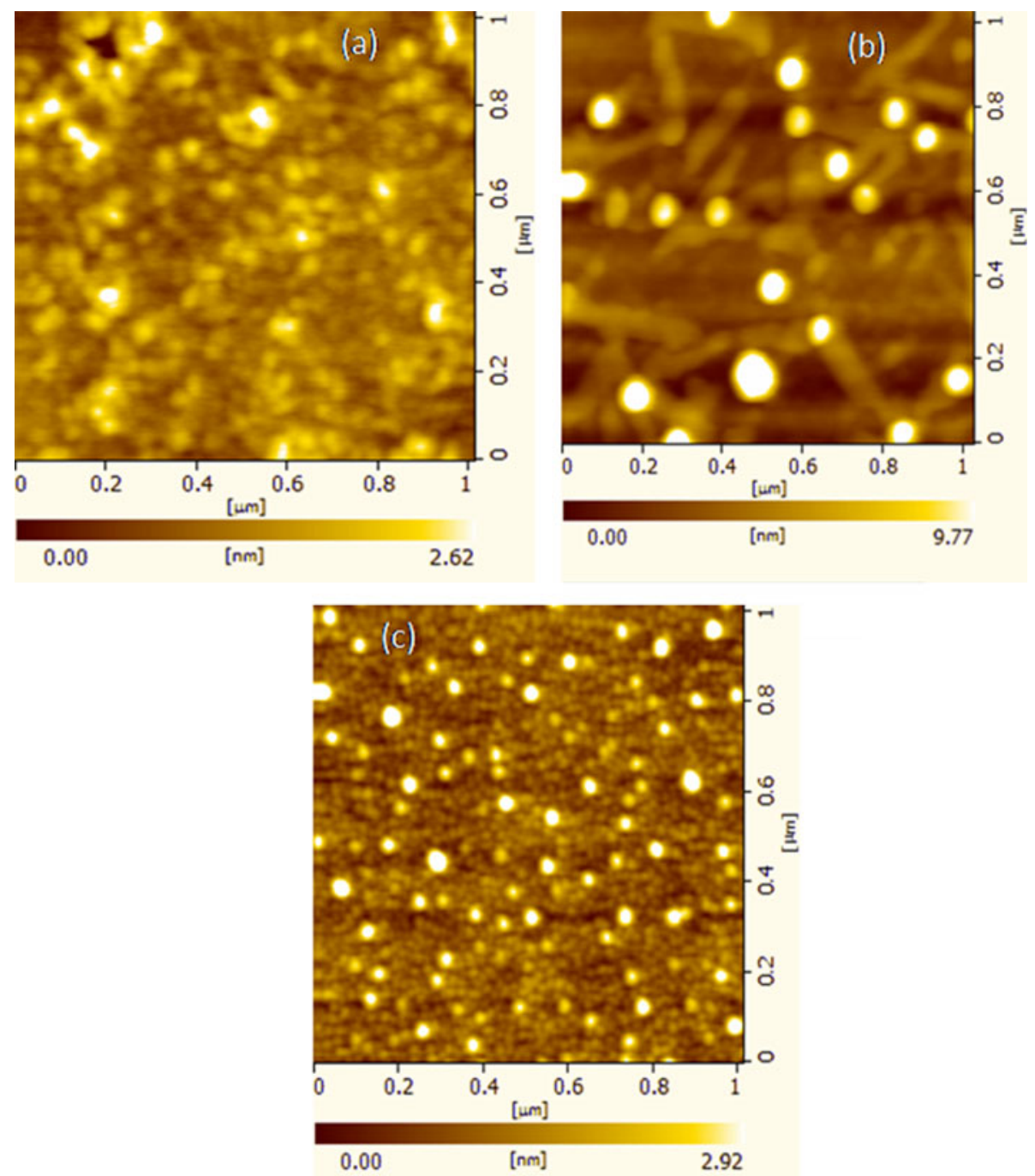

Figure 5. Atomic force images of (a) $50 \mathrm{~nm}$ thick continuous Au film deposited onto BSG substrate and $\mathrm{Au} / \mathrm{Ag}$ bilayer consisting of Au film shown in (a) and an overlayer of Ag deposited on top using an Ar ion energy of $150 \mathrm{eV}$ for (b) 20 and (c) $45 \mathrm{~min}$.

study, on annealing at temperatures $>1173 \mathrm{~K}$ for relatively short times. The beads are of sub-micron rather than nanometric size. However, as in the current study, they have also shown that the formation of beads is dependent on contact angle, surface diffusion length and surface diffusion coefficient. Although this study reports a re-evaporation process, in contrast to the film formation process in the current study, it serves to illustrate the importance of interfacial parameters in determining morphological evolution. Benia et al (2007) reported the growth of $\mathrm{Au}$ islands on $\mathrm{MgO}$ thin films deposited on $\mathrm{Mo}(001)$ surfaces. The authors demonstrate, using STM investigations, the role of $\mathrm{MgO}$ thickness in determining the surface coverage by Au. They have observed similar changes in shape and size of Au nanoparticles as a function of thickness. Gergen et al (2000) demonstrated using AFM images of $\mathrm{Ag}$ and Ti films deposited at room temperature on hydrogen terminated single crystal $\mathrm{Si}$ substrates, that the $\mathrm{Ag}$ films are rough consisting of coalesced islands while the Ti films are relatively smooth. They also observed that the roughness of the Ag films was significantly decreased when Ag was deposited onto a seed layer of Ti predeposited on the Si substrate. A Ti layer of $4 \mathrm{~nm}$ for deposition of $5 \mathrm{~nm}$ of $\mathrm{Ag}$ was sufficient to reduce the Ag film roughness with room temperature deposition. The authors have not elaborated on the reasons for this behaviour. However, from our observations of growth of $\mathrm{Ag}$ and $\mathrm{Au}$ on different interfaces it is evident that the differences in growth habits of $\mathrm{Ag}$ on $\mathrm{Si}$ and $\mathrm{Ag}$ on $\mathrm{Ti} / \mathrm{Si}$ can be attributed to the differences in the interfacial energy and contact angles.

Many theoretical studies on the nucleation and growth of films especially those by Venables and Thompson (Venables et al 1984; Thompson 1990) have shown that the growth of a film on a substrate, up to island formation, is determined by the free energy balance involving the substrate, film and the 

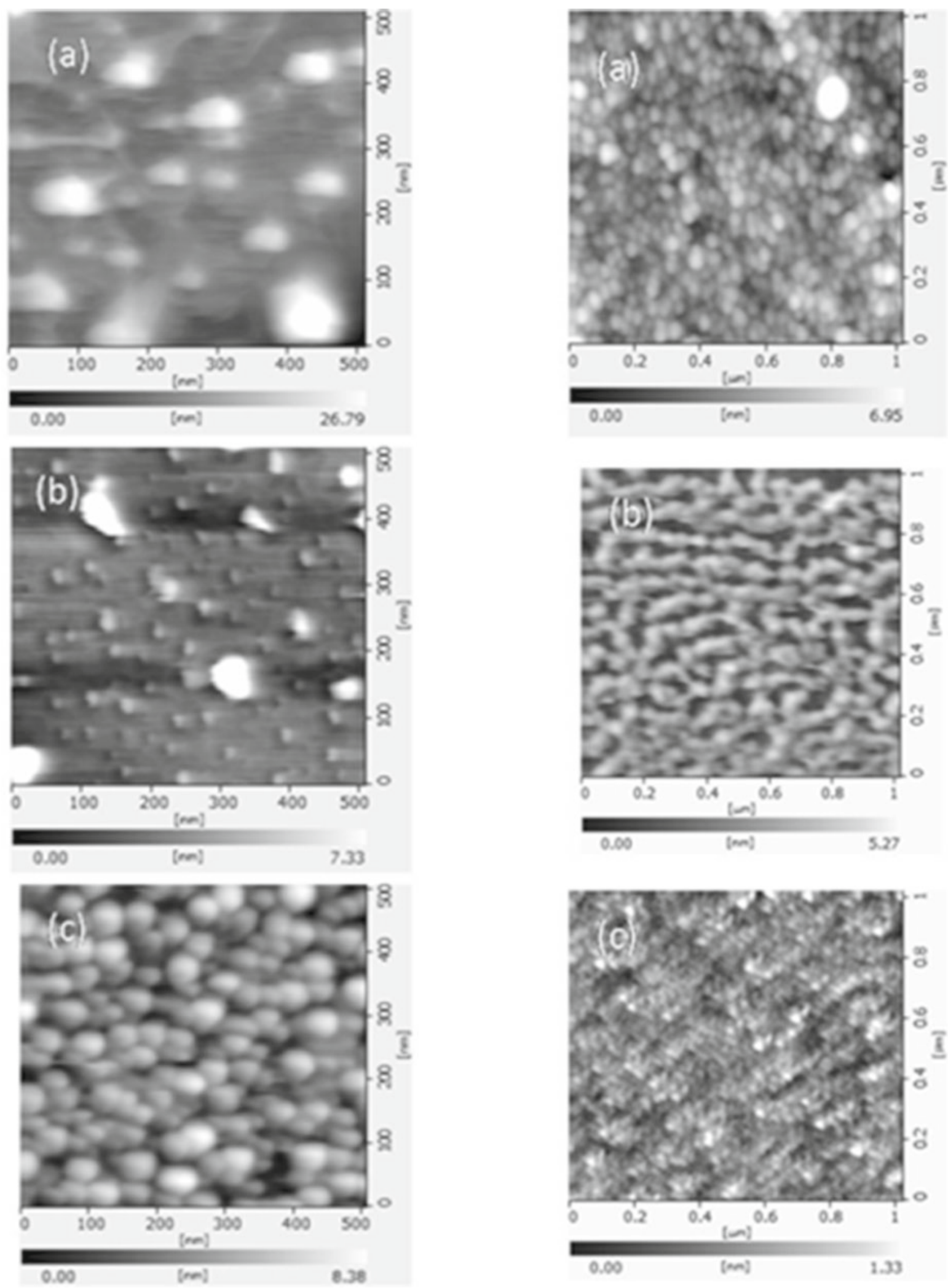

Figure 6. AFM images of $\mathrm{Ag}$ films deposited onto (a) $\mathrm{SiO}_{2}$, (b) $\mathrm{TiO}_{2}$ and (c) $\mathrm{ZrO}_{2}$ films at $150 \mathrm{eV}$ for $20 \mathrm{~min}$.

Figure 7. AFM images of $\mathrm{Ag}$ films deposited onto (a) $\mathrm{SiO}_{2}$, (b) $\mathrm{ZrO}_{2}$ and (c) $\mathrm{TiO}_{2}$ films at $250 \mathrm{eV}$ for $20 \mathrm{~min}$. 

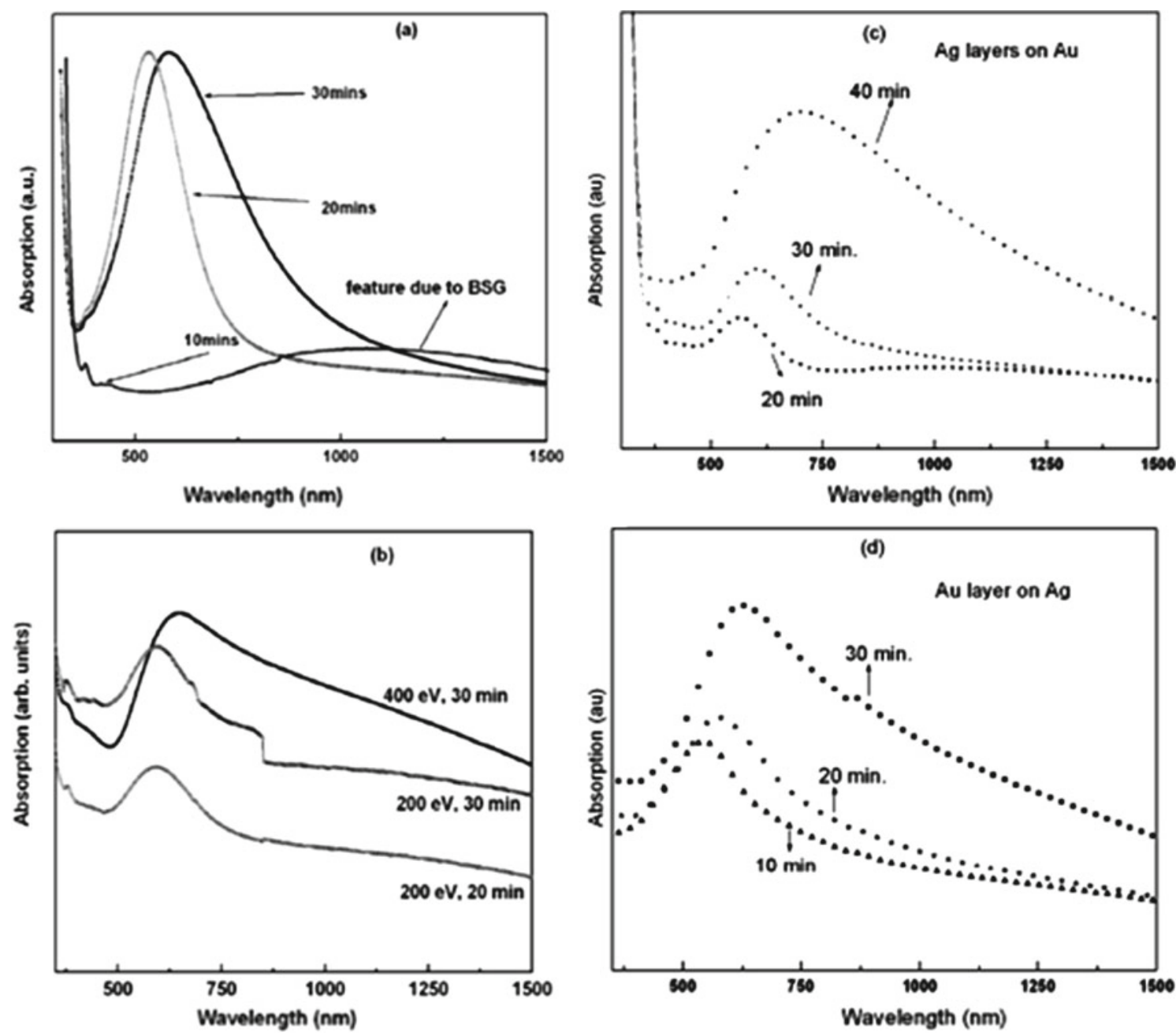

Figure 8. Absorption spectra of (a) Ag and (b) Au films deposited on BSG substrates at different energies and different durations of deposition as indicated, (c) Ag layers at $150 \mathrm{eV}$ on $\mathrm{Au}$ and (d) Au layers at $250 \mathrm{eV}$ on $\mathrm{Ag}$ deposited for different durations of deposition as indicated.

interface between film and substrate. An additional important factor is the contact angle, $\theta$, between film material and substrate. This balance can be expressed as

$$
\gamma_{\mathrm{s}}=\gamma_{\mathrm{i}}+\gamma_{\mathrm{f}} \cos \theta,
$$

where $\gamma_{\mathrm{s}}$ is an excess free energy associated with the substrate surface, $\gamma_{\mathrm{i}}$ the free energy of the island-substrate interface, and $\gamma_{\mathrm{f}}$ the free energy of the film surface. Some materials have a negative enthalpy of mixing with one another-they are miscible. These materials tend to mix strongly at the film substrate interface to give a film-substrate "interface width" that may extend over many atomic layers. Volmer-Weber type of growth leads to 3D islands since the film does not "wet" the substrate and the "interface width" of the growing surface increases with time.

In our experiments, for example, $\gamma_{\mathrm{f}}$ is constant depending on whether the film material is either $\mathrm{Ag}$ or $\mathrm{Au}$. The three control variables would then be $\gamma_{\mathrm{s}}, \gamma_{\mathrm{i}}$ and $\theta$. It is evident from (1) that $\gamma_{\mathrm{s}}$ is determined by the choice of substrate which in turn determines the contact angle, $\theta$. For large contact angles the free energy balance is determined mainly by the free energy of the film-substrate interface. Similarly, for small contact angles the wetting process will determine shape of the particles in the early stages of growth. There will be a similar equilibrium force balance for the free surfaces and grain boundaries.

The average grain size at impingement, defined as

$$
r_{\mathrm{i}}=(A / \pi)^{1 / 2},
$$

where $A$ is the average in-plane area, depends on the relative rates of island nucleation, $N$ the number per unit area per unit time and growth, $L$ the length/time. The growth rate is also governed by the distance, $\delta$, over which atoms adsorbed on the substrate surface can diffuse to a growing island, which depends on the adatom diffusivity on the substrate surface, $D$. The adatom diffusivity has exponential temperature dependence. While the nucleation rate is not a direct function of $\delta$, the fraction of the substrate available for 


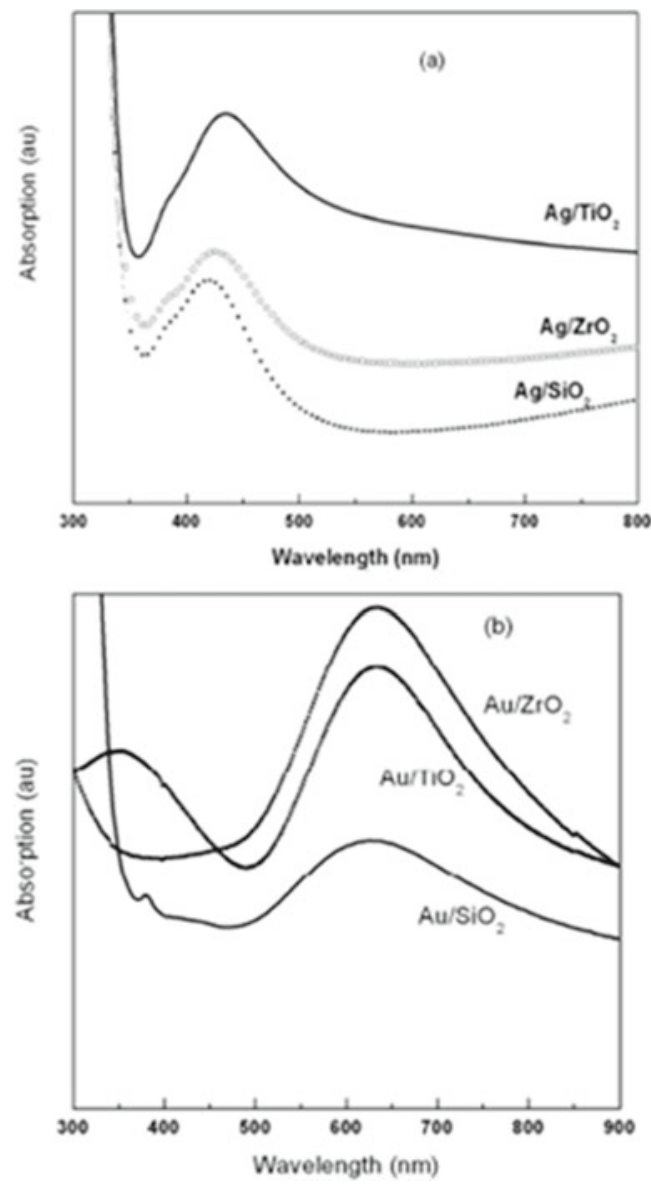

Figure 9. Absorption spectra of (a) $\mathrm{Ag}$ and (b) $\mathrm{Au}$ films deposited on different dielectrics as indicated. Ag and Au films were deposited at ion energies of 150 and $250 \mathrm{eV}$, respectively.

nucleation does depend directly on $\delta$. When $D$ is low, $\delta$ is small, and $r_{\mathrm{i}}$ is proportional to $(L / N)^{1 / 3}$. This means that at ambient temperature both the island nucleation rates and the number of sites available for nucleation are very small leading to island formation. The shapes and sizes of these islands are then entirely driven by the interfacial energy. Furthermore, if the process allows for accurate control of growth rate this would lead to a rich variety of nanostructures as demonstrated in the present study. The results presented in this work clearly demonstrate the role of the film-substrate interface in determining the shape and size of nanostructures formed during the early stages of growth of thin films and also a non-lithographic technique to realize nanostructured thin films.

The nature of plasmon resonances of metal nanoparticles embedded in a dielectric matrix or those at the interface of a dielectric surface have been studied theoretically within the framework of Mie and Maxwell-Garnett theories and the effective medium approximation (Cohen et al 1973; Aspnes et al 1980; Sheng 1980). These theories describe modification of the applied electric field by the dipole fields of individual polarizable spherical metallic particles embedded in a dielectric medium and are valid in the size regime where the macroscopic Maxwell's equation can be applied, which is true so long as their dimensions are less than the wavelength of light in the medium. Starting from Clausius-Mosotti equation it can be shown that if $x$ is the volume fraction of the insulator then the dielectric function of the metal, $\varepsilon_{\mathrm{m}}$ and $\varepsilon_{\mathrm{i}}$, is that of the insulators can be expressed in the form

$$
\frac{\varepsilon(\omega)-\varepsilon_{\mathrm{i}}(\omega)}{\varepsilon(\omega)+2 \varepsilon_{\mathrm{i}}(\omega)}=(1-x) \frac{\varepsilon_{\mathrm{m}}(\omega)-\varepsilon_{\mathrm{i}}(\omega)}{\varepsilon_{\mathrm{m}}(\omega)+2 \varepsilon_{\mathrm{i}}(\omega)} .
$$

Equation (3) assumes that the particles are spherical in shape. The non-spherical shape of the particles is taken into account by introducing the depolarization factors, $L_{\mathrm{m}}$, so that the equation becomes

$$
\frac{\varepsilon(\omega)-\varepsilon_{\mathrm{i}}(\omega)}{\varepsilon(\omega)+2 \varepsilon_{\mathrm{i}}(\omega)}=(1-x) \frac{\varepsilon_{\mathrm{m}}(\omega)-\varepsilon_{\mathrm{i}}(\omega)}{L_{\mathrm{m}} \varepsilon_{\mathrm{m}}(\omega)+\left(1-L_{\mathrm{m}}\right) \varepsilon_{\mathrm{i}}(\omega)}
$$

For spheres $L_{\mathrm{m}}=1 / 3$ and in general, $0 \leq L_{\mathrm{m}} \leq 1$.

These equations have been used theoretically to predict the position of the surface plasmon resonance peaks. It can be shown, from these equations, that the position of the plasmon peaks is dependent on the shape, size and separation of nanoparticles as well as the dielectric function of the dielectric matrix (Brahma et al 2011). The plasmon resonances in metal-metal bilayers, in contrast, are explained on the basis of a concept called plasmon hybridization (Jain and El-Sayed 2007; Prodan et al 2003; Prodan and Nordlander 2004). According to this model, the bilayer system can be considered as a two-interface system that supports two distinct plasmon modes, one due to the outer surface and the other is the inner surface cavity mode. These modes couple or hybridize with each other, leading to a splitting into two new modes, which sometimes manifests itself as increased width of the resonance peak, as in the current case. They further show that the antisymmetric combination of these modes results in a blue-shifted plasmon mode, while the symmetric combination results in a lower red-shifted plasmon mode. They symmetric combination of these modes leads to a plasmon resonance that lies in the visible or near-infrared region. The strength of this plasmon interaction thus determines both the position of the resonance and the extent of splitting or broadening. Thus, the absorption spectrum will exhibit features of both metals, depending on relative concentrations and also a broad-band due to the coupling of the aggregated particles. This is evidently the case in the metal bilayers, in the current study, leading to plasmon resonances over a large range of the spectrum from $\sim 400-700 \mathrm{~nm}$.

\section{Conclusions}

In summary, ion beam sputter deposition of discontinuous $\mathrm{Ag}$ and $\mathrm{Au}$ thin films is reported. The films have been deposited on a variety of substrates. Depending on the nature of the film-substrate interface, a variety of nanostructures 
such as non-regular array of nanoparticles, nanoneedles and nanoclusters have been realized. The growth of these nanostructures by control of the film-substrate interface is demonstrated. It is also shown that the varied morphology of the films leads to a wide range of plasmon resonances extending over a wavelength range from $400-700 \mathrm{~nm}$.

\section{Acknowledgements}

One of the authors (RB) acknowledges the Rajiv Gandhi National Fellowship for this work. Facilities provided by the DST sponsored Centre for Nanotechnology and UGC-CAS programme in the School of Physics, are also gratefully acknowledged.

\section{References}

Aspnes D E, Kinsbron E and Bacon D D 1980 Phys. Rev. B21 3290 Balla T, Spearing S M and Monk A 2008 J. Phys. D: Appl. Phys. 41 174001

Benia H M, Lin X, Gao H-J, Nilius N and Freund H-J 2007 J. Phys. Chem. C111 10528

Beszeda I, Kocsis T, Imreh G, Weigl F, Boyen H-G, Ziemann P and Beke D L 2006 Appl. Surf. Sci. 2531160

Brahma R and Krishna M G 2008 Nucl. Instrum. Meth. B266 1493

Brahma R and Krishna M G 2011 Physica E43 1192

Brahma R, Mahipal Reddy G, Anantha Lakshmi P, Singh S and Krishna M G 2011 J. Nanosci. Nanotechnol. 111
Cohen R W, Cody G D, Coutts M D and Abeles B 1973 Phys. Rev. B8 3689

Didiot C, Pons S, Kierren B, Fagot-Revurat Y and Malterre D 2007 Nat. Nanotech. 2617

Gamo K 1997 Nucl. Instrum. Meth. B121 464

Gergen B, Nienhaus H, Weinberg W H and McFarland E M 2000 J. Vac. Sci. Technol. B18 2401

Jain P K and El-Sayed M A 2007 Nano Lett. 72854

Krishna M G and Kumar P 2009 in Emerging nanotechnologies for manufacturing (eds) M Jackson and W Ahmed (Massachussetts, USA: William Andrew, Elsevier Imprint) p. 93

Maissel L and Glang R 1970 in Handbook of thin film technology (McGraw Hill, New York, USA)

Masala O and Seshadri R 2004 Ann. Rev. Mater. Res. 3441

Prodan E and Nordlander P 2004 J. Chem. Phys. 1205445

Prodan E, Radloff C, Halas N J and Nordlander P 2003 Science 302419

Rao C N R et al 2006 Pure Appl. Chem. 781619

Sheng P 1980 Phys. Rev. Lett. 4560

Siegel J, Lyutakov O, Rybka V, Kolská Z and Švorčík V 2011 Nanosci. Res. Lett. 696

Stangl J, Holý V and Bauer G 2004 Rev. Mod. Phys. 76725

Teichert C 2002 Phys. Rep. 365335

Tennant D M and Bleier A R 2011 in Comprehensive nanoscience and technology (New York: Academic Press, Elsevier Imprint) 4 p. 35

Thompson C V 1990 Ann. Rev. Mater. Sci. 20245

Venables J A, Spiller G D T and Hanbucken M 1984 Rep. Prog. Phys. 47399

Woll A R, Rugheimer P and Lagally M G 2002 Mater. Sci. Eng. B96 94

Zhang E Y and Wang C R 2009 Curr. Opin. Coll. Interf. Sci. 14148 